\title{
Error-corrected sequencing strategies enable comprehensive detection of leukemic mutations relevant for diagnosis and minimal residual disease monitoring
}

Erin L. Crowgey ${ }^{1}$, Nitin Mahajan ${ }^{2,3}$, Wing Hing Wong ${ }^{2,3}$, Anilkumar Gopalakrishnapillai', Sonali P. Barwe ${ }^{1}$, E. Anders Kolb ${ }^{4}$ and Todd E. Druley ${ }^{2,3^{*}}$

\begin{abstract}
Background: Pediatric leukemias have a diverse genomic landscape associated with complex structural variants, including gene fusions, insertions and deletions, and single nucleotide variants. Routine karyotype and fluorescence in situ hybridization (FISH) techniques lack sensitivity for smaller genomic alternations. Next-generation sequencing (NGS) assays are being increasingly utilized for assessment of these various lesions. However, standard NGS lacks quantitative sensitivity for minimal residual disease (MRD) surveillance due to an inherently high error rate.
\end{abstract}

Methods: Primary bone marrow samples from pediatric leukemia $(n=32)$ and adult leukemia subjects $(n=5)$, cell line MV4-11, and an umbilical cord sample were utilized for this study. Samples were sequenced using molecular barcoding with targeted DNA and RNA library enrichment techniques based on anchored multiplexed PCR (AMP®) technology, amplicon based error-corrected sequencing (ECS) or a human cancer transcriptome assay.

Computational analyses were performed to quantitatively assess limit of detection (LOD) for various DNA and RNA lesions, which could be systematically used for MRD assays.

Results: Matched leukemia patient samples were analyzed at three time points; diagnosis, end of induction (EOI), and relapse. Similar to flow cytometry for ALL MRD, the LOD for point mutations by these sequencing strategies was $\geq 0.001$. For DNA structural variants, FLT3 internal tandem duplication (ITD) positive cell line and patient samples showed a LOD of $\geq 0.001$ in addition to previously unknown copy number losses in leukemia genes. ECS in RNA identified multiple novel gene fusions, including a SPANT-ABL gene fusion in an ALL patient, which could have been used to alter therapy. Collectively, ECS for RNA demonstrated a quantitative and complex landscape of RNA molecules with $12 \%$ of the molecules representing gene fusions, $12 \%$ exon duplications, $8 \%$ exon deletions, and $68 \%$ with retained introns. Droplet digital PCR validation of ECS-RNA confirmed results to single mRNA molecule quantities.

Conclusions: Collectively, these assays enable a highly sensitive, comprehensive, and simultaneous analysis of various clonal leukemic mutations, which can be tracked across disease states (diagnosis, EOI, and relapse) with a high degree of sensitivity. The approaches and results presented here highlight the ability to use NGS for MRD tracking.

Keywords: Error-corrected sequencing, Minimal residual disease, Next generation sequencing, Pediatric leukemia, Computational biology

\footnotetext{
* Correspondence: druley_t@wustl.edu

2Department of Pediatrics, Washington University School of Medicine, 660

South Euclid Avenue, Campus Box 8116, St. Louis, MO 63110, USA

${ }^{3}$ Edison Family Center for Genome Sciences and Systems Biology,

Washington University School of Medicine, 4515 McKinley Avenue, Campus

Box 8510, St. Louis, MO 63108, USA

Full list of author information is available at the end of the article
}

(c) The Author(s). 2020 Open Access This article is distributed under the terms of the Creative Commons Attribution 4.0 International License (http://creativecommons.org/licenses/by/4.0/), which permits unrestricted use, distribution, and reproduction in any medium, provided you give appropriate credit to the original author(s) and the source, provide a link to the Creative Commons license, and indicate if changes were made. The Creative Commons Public Domain Dedication waiver (http://creativecommons.org/publicdomain/zero/1.0/) applies to the data made available in this article, unless otherwise stated. 


\section{Background}

Genetic characterization of leukemias is a critical component of the clinical evaluation, risk stratification, and therapeutic strategy [1]. Chromosomal rearrangements generating gene fusions and complex structural variants (StVs) are more common in childhood than in adult leukemias (M. J [2].). Recently, genomic data analysis of cancer patients has highlighted the significance of these genomic rearrangements and single nucleotide variants (SNVs), causing an increase in the use of site-specific qPCR, RT-qPCR or gene expression profiling for diagnostic characterization (M. J [3].). However, leukemias are heterogenous at the genomic level, with many rare variants driving clinically indistinguishable disease. Single variant assays require a priori knowledge of the mutations present, thus limiting the scalability of such an approach.

Minimal residual disease (MRD; more aptly named Measurable Residual Disease) is the detection of residual leukemia following therapy, most commonly by flow cytometry. MRD, as measured by multiparameter flow cytometry is perhaps one of the most important predictors of outcome in children with acute myelogenous leukemia (AML) [4]. For pediatric acute lymphoblastic leukemia (ALL) flow cytometry data, with a limit of detection of 0.0001 for clonal B- or T-cell surface receptors, the "leukemia-associated immunophenotype" (LAIP), has been shown to correlate with significantly worse disease-free and overall survival (M. J [3].; M. J [2].).

In contrast, because myeloid cells do not harbor a single clonal surface marker, multi-parameter flow cytometry (MPFC) for a profile of surface immunophenotypes displaying a "different from normal" cell population is the current gold standard for MRD in AML with a limit of detection of approximately 0.001 [4]. However, surface immunophenotypes often change during AML therapy [5]. Moreover, MPFC does not enable identification of genomic lesions that are associated with treatment options and risk stratification.

Standard NGS platforms have systematic error rates of approximately 0.005-0.02 (A. L. [6]; A. L. [7]), depending on the platform and analytic strategy. Thus, NGS strategies are limited in their ability to detect variants at allele frequencies below the error rate of the platform, plus the sequencing depth requirements for bulk sequencing to achieve these limits of detection are cost prohibited. Error corrected sequencing (ECS) mitigates systematic errors via the incorporation of a unique molecular index (UMI) to each molecule captured (A. L. [6]) allowing for errors to be discarded and very rare mutations to be clearly detected in heterogeneous nucleic acid samples. Furthermore, by coupling UMIs with anchored multiplexed PCR (AMP) technology, the robust identification of $\mathrm{StVs}$ in DNA, including copy number variations, or RNA, including cryptic gene fusions, is enabled.
Adding UMIs across an entire genome or transcriptome is cost prohibitive, and there is no "one size fits all" strategy for detecting and tracking the wide variety of mutation in any given cancer. In this study, our goals were to demonstrate various quantitative strategies for comprehensive detection of complex, low frequency mutations from a collection of leukemia samples with known diagnostic results and outcomes. The data presented demonstrate the ability to create NGS strategies capable of reaching limits of detection appropriate for MRD. Furthermore, the results highlight the robust ability of ECS to quantitatively assess the complex mutational landscape of leukemias, which will facilitate precision therapeutic selection and ultimately reduced morbidity and improved survival.

\section{Methods}

Samples and consent

All samples used in this study were collected from human peripheral blood or bone marrow. Specimens were collected for biobanking and subsequent de-identified biomedical research at Nemours following written informed consent $(\mathrm{Hu}-$ man Research Protection Office (HRPO) IRB\# 349465), including parental permission forms (child/adolescent assent was obtained for ages 7-17 years). The Nemours samples used in this study were deemed non-human research and approved by the Nemours / A.I. duPont Children's Hospital (HRPO IRB\# 267207). Patients enrolled on the Children's Oncology Group (COG) AAML1031 phase III pediatric de novo AML study (NCT00372593) had the option to consent/assent for correlative biomedical research when enrolling for the study. All available COG samples were de-identified and personal health information (PHI) was not available to study team. Adult AML specimens are banked at Washington University for biomedical research under Human Research Protection Office (HRPO) IRB\# 201011766. This study was approved as by the Washington University HRPO IRB\# 201511125 entitled "Error-corrected sequencing for minimal residual disease in AML".

Samples from Washington University included an umbilical cord sample and adult $(n=5)$ de novo AML samples with known FLT3-ITD lesions. COG samples were collected from pediatric de novo AML patients enrolled on the COG Phase III prospective study, AAML1031. In total we sequenced 4 unique subjects, with each subject having 2 or 3 timepoints collected (diagnosis, end of induction (EOI), relapse). The Nemours samples consisted of six AML subjects, one acute promyelocytic leukemia (APL) subject, 17 preB-cell ALL subjects and three Tcell ALL subjects (Table 1). Demographic data for these samples are summarized in Additional file 5: Table S1. The sample distribution matches with the incidence rate of pediatric AML, APL, B-ALL and TALL reported in US population, respectively. 
Table 1 Summary of variants and allele frequencies in longitudinal samples

\begin{tabular}{|c|c|c|c|c|c|c|c|c|}
\hline Assay & Sample & Gene & AF Diagnosis & AFEOI & AF Relapse & Position & Ref / Alt & Effect \\
\hline \multirow{3}{*}{ RNA } & \multirow{3}{*}{ COG Subject 1} & FOXP1 & 0.115 & 0.0609 & 0.115 & chr3:71349029 & $\mathrm{A} / \mathrm{AC}$ & splice_region_variant\&5_prime_UTR_variant \\
\hline & & NOTCH1 & 0.2453 & 0.119 & 0.2453 & chr9:139396940 & $\mathrm{C} / \mathrm{CT}$ & frameshift_variant\&splice_region_variant \\
\hline & & MLF1 & 0.9939 & 0.75 & 0.9509 & chr3:158289136 & $\mathrm{CG} / \mathrm{C}$ & splice_donor_variant \\
\hline \multirow{5}{*}{ RNA } & \multirow{5}{*}{ COG Subject 2} & EIF4A1 & 0.875 & 0.36 & 0.2941 & chr17:7479907 & CACCA / TACCC & missense_variant \\
\hline & & CEBPA & 0.4476 & 0 & 0.3867 & chr19:33792381 & C/CCTTGTCGGTGTCCTT & inframe_insertion \\
\hline & & CEBPA & 0.4718 & 0 & 0.4009 & chr19:33793133 & T/TAGCGATGTAGG & frameshift_variant \\
\hline & & KAT6A & 0.85 & 0.625 & 0.8594 & chr8:41798962 & $\mathrm{C} / \mathrm{CA}$ & frameshift_variant\&splice_region_variant \\
\hline & & IKZF1 & 0.078 & 0.3475 & 0.2246 & chr7:50367354 & G/A & missense_variant \\
\hline \multirow{7}{*}{ DNA } & \multirow{7}{*}{ COG Subject 3} & WT1 & 0.4459 & 0.0026 & 0.4109 & chr11:32449576 & $\mathrm{C} / \mathrm{CG}$ & frameshift_variant \\
\hline & & NPM1 & 0.3815 & 0.001 & 0.3196 & chr5:170837545 & C/CTGCA & frameshift_variant \\
\hline & & GATA2 & 0.4383 & 0.3287 & 0.4292 & chr3:128205837 & $\mathrm{T} / \mathrm{G}$ & missense_variant \\
\hline & & RUNX1 & 0.229 & 0.1322 & 0.2244 & chr21:36206758 & $\mathrm{A} / \mathrm{C}$ & missense_variant \\
\hline & & RUNX1 & 0.464 & 0.3817 & 0.5062 & chr21:36206770 & $\mathrm{T} / \mathrm{G}$ & missense_variant \\
\hline & & TET2 & 0.2402 & 0.1628 & 0.2047 & chr4:106196310 & $\mathrm{A} / \mathrm{C}$ & missense_variant \\
\hline & & SRSF2 & 0.1338 & 0.064 & 0.1423 & chr17:74732362 & $A / C$ & missense_variant \\
\hline \multirow{5}{*}{ DNA } & \multirow{5}{*}{ COG Subject 4} & ASXL1 & 0.2127 & 0.0298 & relapse* & chr20:31022441 & $\mathrm{A} / \mathrm{AG}$ & frameshift_variant \\
\hline & & SETD2 & 0.0433 & 0.0193 & relapse* & chr3:47163976 & $G / C$ & stop_gained \\
\hline & & PTPN11 & 0.0281 & 0.0071 & relapse* & chr12:112924366 & $\mathrm{T} / \mathrm{C}$ & missense_variant \\
\hline & & CREBBP & 0.0259 & 0.005 & relapse* & chr16:3820818 & $\mathrm{G} / \mathrm{C}$ & missense_variant \\
\hline & & CREBBP & 0.0261 & 0.0053 & relapse* & chr16:3820821 & $\mathrm{A} / \mathrm{C}$ & missense_variant \\
\hline
\end{tabular}

\section{Nucleic acid extraction}

Nucleic acid was extracted from each sample in the Druley laboratory at Washington University. DNA was extracted using the Qiagen (Germantown, Maryland, USA) DNeasy Blood \& Tissue Kit (catalog \#: 69504) using the manufacturer's protocol. RNA was extracted using the Qiagen RNeasy Plus Mini Kit (catalog \#: 74134). Nucleic acid quantity and quality was then assessed using the Agilent (Santa Clara, California USA) TapeStation 4200 following the manufacturer's protocol using the High Sensitivity D1000 Screen Tape (catalog \#: 5067-5584) for DNA or the High Sensitivity RNA Screen Tape (cata$\log \#$ : 5067-5579) for RNA.

\section{DNA-ECS library preparation}

To optimize detection of structural and copy number variants in DNA and RNA in genes more closely affiliated with pediatric leukemia, we prepared DNA-ECS libraries using a customized ArcherDx (Boulder, Colorado USA) VariantPlex kit based on published data from the Therapeutically Applicable Research to Generate Effective Treatments (TARGET) project conducted by COG [8]. The custom assay consists of 1395 primer pairs and required $250 \mathrm{ng}$ of DNA for input. Primer amplification uniformity was $>95 \%$ for the all assays.

\section{RNA-ECS library preparation}

There were two different RNA-ECS library preparation methods used in this study. First, for absolute quantification of transcript copy number in 416 human cancer related genes, we adapted the Qiagen Human Cancer Transcriptome kit (catalog \#: RHS-003Z) to be compatible with our UMI-aware bioinformatics, similar to our published DNA method (A.L. [7]). Total RNA was extracted using RNeasy Mini Kit (Qiagen, Inc), and cDNA was made from $50 \mathrm{ng}$ of RNA using the QIAseq kit. Each cDNA molecule from the region of interest was then tagged with an UMI prior to PCR amplification. Libraries were made using the QIAseq kit following the manufacturer's protocol. For each sample, a technical replicate was also made. Second, for quantitative characterization of structural variation in mRNA (fusions, aberrant splice isoforms, retained introns), the ArcherDX FusionPlex HemeV2 Kit (catalog \#: AB0012) was utilized per manufacturer's protocols.

\section{Bioinformatics}

The data analysis for the ArcherDx library preparation followed the following steps: read quality cleaning, error correction, genome alignment, and variant detection and annotation. Fastq files were analyzed via FASTQC for quality, and trimmed based on adaptor sequences and quality. Trimmed reads were aligned to hg19 using bwa mem, bowtie2, and mummer3, whereas de novo reference assembly was conducted by velvet and AMRA (Additional file 1: Figure S1). SNVs and short InDels $(\leq 20 \mathrm{bp}$ ) were detected from the genomic alignments by freeBayes and Lofreq, whereas large InDels (> $20 \mathrm{bp}$ ) were detected via custom de novo assembly. Variants were filtered based on depth of error-corrected sequencing bins, minimum of 3 , that supported the call. All regions in which variants were called required a total read depth $>100 \mathrm{X}$, and a minimal base quality score (phred) of 20 was applied. The ExAC database was used to annotate common variants. Alignment files were processed through several algorithms for variant detection. Data analysis was conducted using an Amazon Web Service that hosted the ArcherDx Analysis platform (v5.1.3). 


\section{Copy number variation analysis}

CNV filtering was based on the following: 1) a $p$-value calculation, based on a two-tailed Wilcoxon rank sum test, with a null hypothesis that the median value of the copy number called for each probe of the given target was equal to the median value of the copy number called for all primers identified as being in the baseline, across all samples analyzed; 2) standard deviation of the called copy number for all probes of the target; 3 ) total number of adjacent gene-specific primers (GSPs) supporting the CNV event; and 4) variants deemed likely to be a chemistry or sequencing artifact were filtered out.

\section{Variant allele determination for complex variants}

The variant allele frequency (VAF) for a FLT3 internal tandem duplication (FLT3-ITD) is calculated by comparing reads supporting the wild type (WT) FLT3 junction in that region, to the reads supporting a novel junction. To optimize detection of variably sized duplications/deletions, the small ITD $(<20 \mathrm{bp})$ are detected via forced reference alignment, and the larger ( $>20 \mathrm{bp}$ ) ITD are detected via de novo assembly, as highlighted in Additional file 1: Figure S1. It is essential to consider the following characteristics of the assay: read depths, sample complexity, depth of coverage, breadth of coverage, molecular bin sizes, and power analysis to detect low allelic variants. We analyzed the following: (1) coverage per targeted base at different read depths, (2) coverage versus error correctable coverage for all targeted bases 35 million and full depth, (3) percentage of targeted bases covered at $35 \mathrm{M}$ reads per sample, (4) percent of targeted bases covered at various read depths (5) statistical power to call low allelic variants based on background noise at that position and cohort level sequencing metrics (6) summary of error correctable coverage and sensitivity for all targeted bases at individual sample level.

For RNA-ECS, raw sequencing reads sharing the same UMIs were aligned to each other to form read families as per DNA-ECS analysis. A minimum of three reads per UMI was required for the downstream process of de-duplication and error-correction. A consensus read was then made for each read family and aligned to hg19.

\section{Results}

\section{Application of ECS for MRD}

To assess the limits of detection (LOD) in a clinically meaningful scenario, we retrospectively applied our custom DNA- and RNA-ECS assays to longitudinal pediatric leukemia samples collected from four unique subjects. Each subject had 2 or 3 timepoints collected (diagnosis, EOI, relapse). COG subject 3 had all time points analyzed, diagnosis, EOI, and relapse, and the results are demonstrated in Fig. 1. The top panel in Fig. 1 represents the analysis between diagnosis and EOI, with the left axis and blue lines representing VAF across the

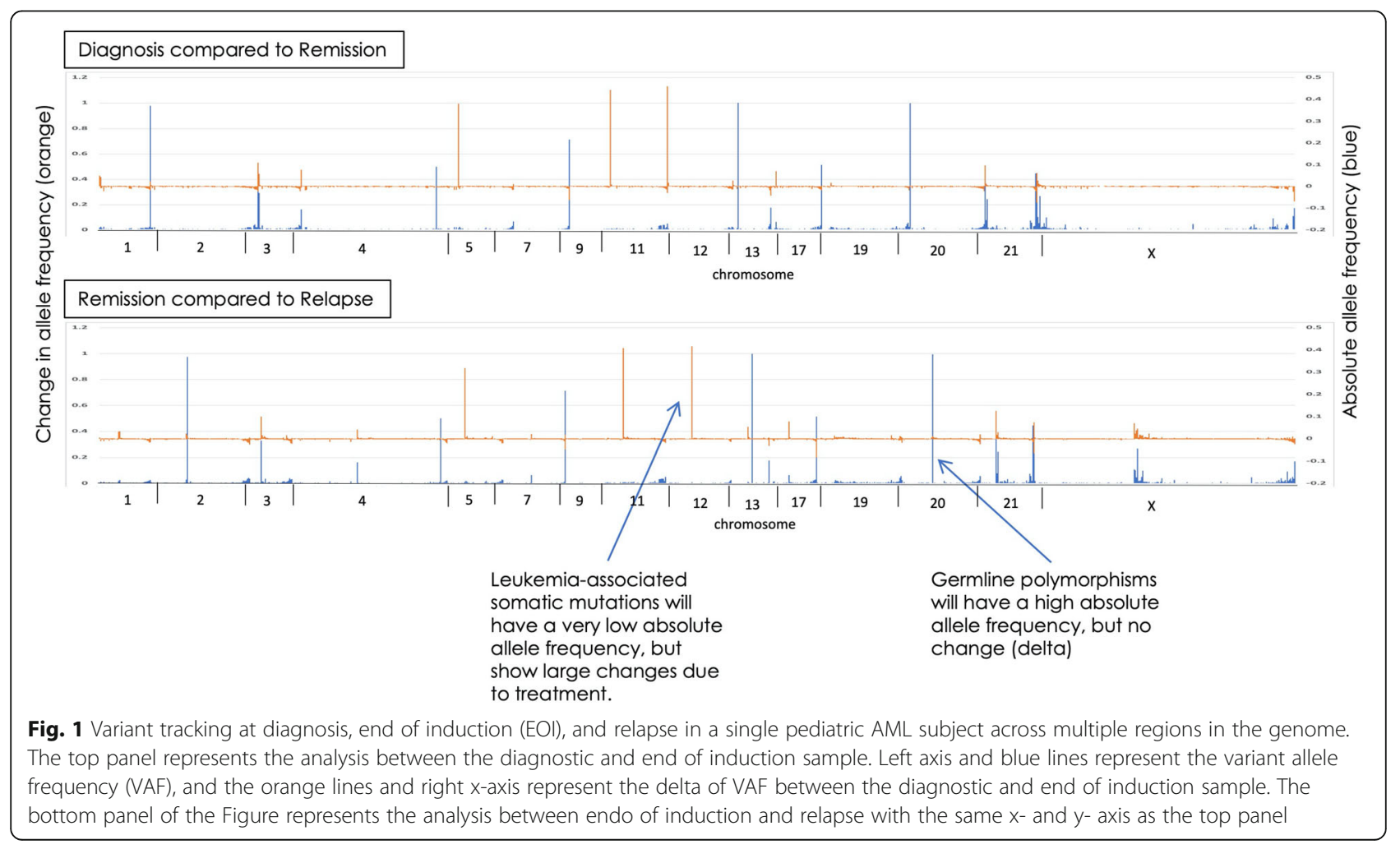


genome, and the orange lines and right $\mathrm{x}$-axis representing the delta, or change, in VAF between diagnosis and EOI. The data demonstrate the detection of noise and disease relevant variations. The bottom panel in Fig. 1 represents the analysis between EOI and relapse. Using the EOI sample to help annotate germline variants, in total 40 germline mutations were detected, 5 mosaic like variants, and 4 somatic variants were tracked across all 3 samples: PTPN11, WT1, NPM1, and FLT3-ITD. Each of these variants were detected at EOI using error-corrected sequencing reads, with VAF ranging from $0.001-0.0026$, which are frequencies below the LOD for NGS without molecular indexing and consistent with leukemic cell LOD by flow cytometry for MRD. ECS provides noise reduction that permits detection of variants with low allele frequencies that are below the limit of detection by bulk sequencing strategies (WES data from TARGET). Figure 2 highlights the ECS background noise reduction (blue dots) for the PTPN11 variant (red dot) identified at a low allelic frequency after induction therapy.

Table 1 summarizes the variants identified in the 4 subjects analyzed. Of interest, COG subject 2 had an IKZF1 mutation at low allelic frequency at time of diagnosis (0.078), which increased at both the EOI (0.3475) and relapse state (0.2246). COG subject 1 had a NOTCH1 and FOXP1 splice region variants that were detectable at EOI (Table 2). COG subject 4 relapsed, but the sample was not available for analysis and only the diagnostic and EOI samples have been analyzed, which indicated several potential variants of interest for disease tracking (Table 1).

\section{Limit of detection (LOD) for FLT3-ITD}

To analyze the limit of detection (LOD) for complex variants, we focused on FLT3-ITD in DNA (Additional file 6: Table S2). To determine the LOD, DNA from a human leukemia cell line (MV4-11) with a 30 bp FLT3-ITD was serially diluted into human genomic DNA followed by DNA-ECS. The $30 \mathrm{bp}$ FLT3-ITD was detected in all samples at expected frequencies (Fig. 3a). Given the variable size of FLT3-ITDs, from a few bases to hundreds, we utilized primary bone marrow samples from AML subjects $(n=9)$ with known FLT3-ITDs to establish a range of allele detection frequencies and sizes. DNA-ECS detected all of the ITDs across different sizes (range 3-90 bp) and frequencies (0.0001-0.43) as shown in Fig. 3b. These data further demonstrate the ability to detect FLT3-ITD mutations with molecular diagnostics at a LOD appropriate for MRD detection.

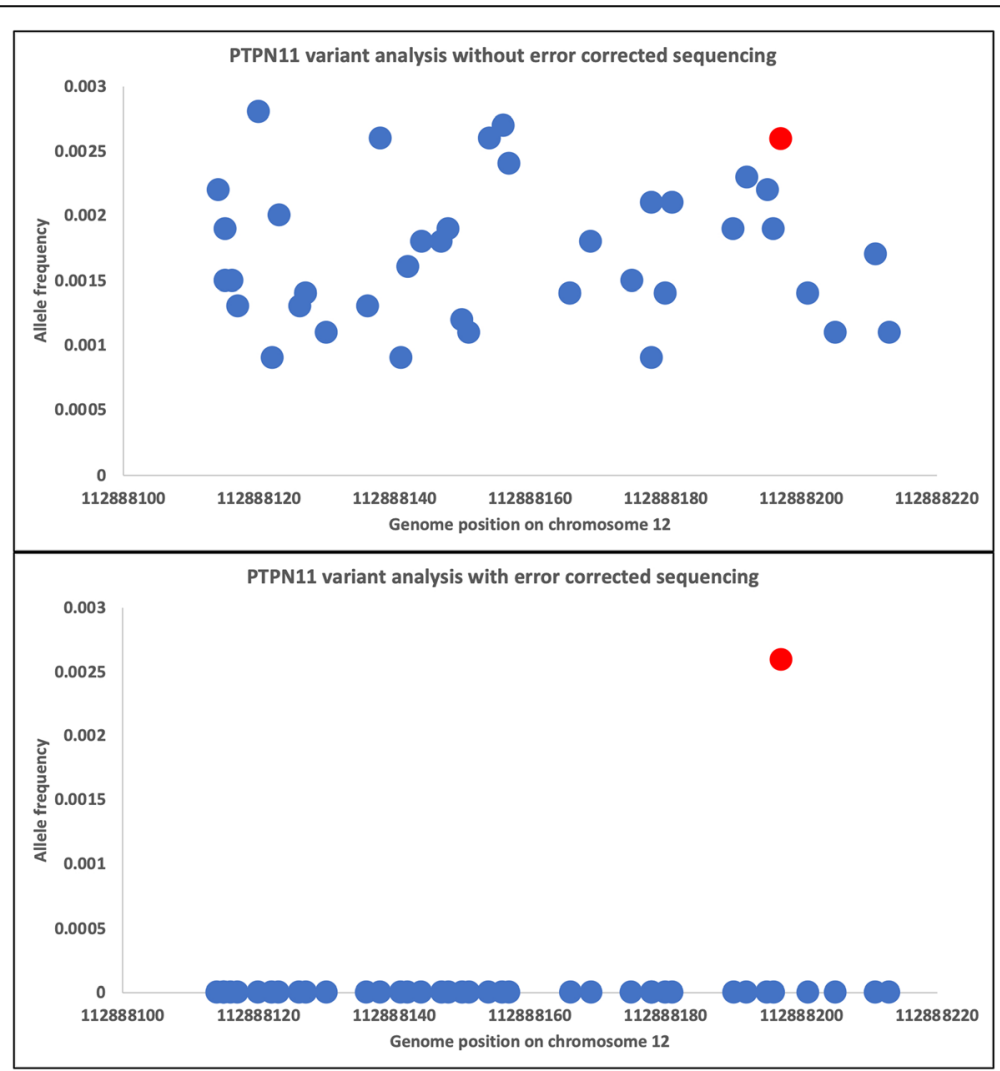

Fig. 2 Error-corrected sequencing noise reduction for low allelic variants. Top panel represents all variants (blue dots) identified in the genomic region of a known somatic mutation located in PTPN11 (red dot). Bottom panel demonstrates noise reduction with the application of error-corrected sequencing 
Table 2 Comparison between gene rearrangements detected by diagnostic FISH analysis and by targeted error-corrected sequencing of pediatric primary bone marrow specimens

\begin{tabular}{|c|c|c|}
\hline Leukemia & Diagnosis with FISH & Fusions \\
\hline \multirow{6}{*}{ AML } & $\begin{array}{c}\text { AML } \\
\text { deletion of } 5 q \text { and } 7 q\end{array}$ & none \\
\hline & $\begin{array}{c}\text { DS-AML } \\
\text { Low level trisomy } 8 \\
\end{array}$ & none \\
\hline & $\begin{array}{c}\text { AML } \\
\text { MLL rearrangement }\end{array}$ & KMT2A - MLLT3 (56\%) \\
\hline & $\begin{array}{c}\text { AML } \\
\text { MLL-ENL, RUNX1T1 extra signal detected }\end{array}$ & KMT2A- MLLT1 (51\%) \\
\hline & $\begin{array}{c}\text { AML } \\
\text { normal }\end{array}$ & P2RY8 - CRLF2 (0.1\%) \\
\hline & $\begin{array}{c}\text { AML } \\
\text { normal }\end{array}$ & NUP98 - NSD1 (20\%); NSD1-NUP98 (7\%) \\
\hline APL & $\begin{array}{c}\text { APL } \\
\text { PML-RARA fusion }\end{array}$ & PML - RARA (20\%) \\
\hline \multirow{20}{*}{ ALL } & $\begin{array}{c}\text { B-ALL } \\
\text { BCR/ABL1 translocation } \\
\end{array}$ & BCR-ABL (56\%) \\
\hline & $\begin{array}{c}\text { B-ALL } \\
\text { E2A gene deletion }\end{array}$ & TCF3 - PBX1 (20\%) \\
\hline & $\begin{array}{c}\text { B-ALL } \\
\text { E2A/PBX1 translocation }\end{array}$ & TCF3 - PBX1 (52\%) \\
\hline & $\begin{array}{c}\text { B-ALL } \\
\text { ETV6/RUNX1 fusion, MLL gene deletion }\end{array}$ & ETV6- RUNX1 (31\%) \\
\hline & $\begin{array}{c}\text { B-ALL } \\
\text { ETV6/RUNX1 fusion, p16 gene deletion }\end{array}$ & ETV6- RUNX1 (8\%) \\
\hline & $\begin{array}{c}\text { B-ALL } \\
\text { ETV6/RUNX1 translocation }\end{array}$ & ETV6- RUNX1 (44\%) \\
\hline & $\begin{array}{c}\text { B-ALL } \\
\text { ETV6/RUNX1 translocation }\end{array}$ & $\begin{array}{c}\text { ETV6- RUNX1 (56\%); P2RY8 - CRLF2 (0.6\%); RUNX1- } \\
\text { MKL1 (50\%) }\end{array}$ \\
\hline & $\begin{array}{c}\text { B-ALL } \\
\text { hyperdiploidy, p16 gene deletion }\end{array}$ & none \\
\hline & $\begin{array}{c}\text { B-ALL } \\
\text { hyperdiploidy, trisomy } 4,10 \text {, tetrasomy } 21\end{array}$ & P2RY8 - ANKLE2 (18\%) \\
\hline & $\begin{array}{c}\text { B-ALL } \\
\text { normal }\end{array}$ & none \\
\hline & $\begin{array}{c}\text { B-ALL } \\
\text { normal }\end{array}$ & IKFF1 exon 4-6 deletion \\
\hline & $\begin{array}{c}\text { B-ALL } \\
\text { normal }\end{array}$ & SSBP2- CHD1 (12\%); P2RY8 - CRLF2 (0.3\%) \\
\hline & $\begin{array}{l}\text { B-ALL } \\
\text { normal }\end{array}$ & TCF3 - HLF (23\%) \\
\hline & $\begin{array}{c}\text { B-ALL } \\
\text { p16 gene deletion }\end{array}$ & IKZF1 exon 4-6 deletion (44\%) \\
\hline & $\begin{array}{c}\text { B-ALL } \\
\text { p16 gene deletion }\end{array}$ & none \\
\hline & $\begin{array}{c}\text { B-ALL } \\
\text { RUNX1 amplification }\end{array}$ & RCAN1 - RUNX1 (1\%); P2RY8 - CRLF2 (0.6\%) \\
\hline & $\begin{array}{c}\text { B-ALL } \\
\text { RUNX1 amplification } \\
\end{array}$ & P2RY8 - CRLF2 (73\%) \\
\hline & $\begin{array}{c}\text { T-ALL } \\
\text { normal }\end{array}$ & none \\
\hline & $\begin{array}{c}\text { T-ALL } \\
\text { normal }\end{array}$ & SPTAN1 - ABL1 (56\%); STIL - TAL1 (30\%) \\
\hline & $\begin{array}{l}\text { T-ALL } \\
\text { normal }\end{array}$ & STIL - TAL1 (42\%) \\
\hline
\end{tabular}




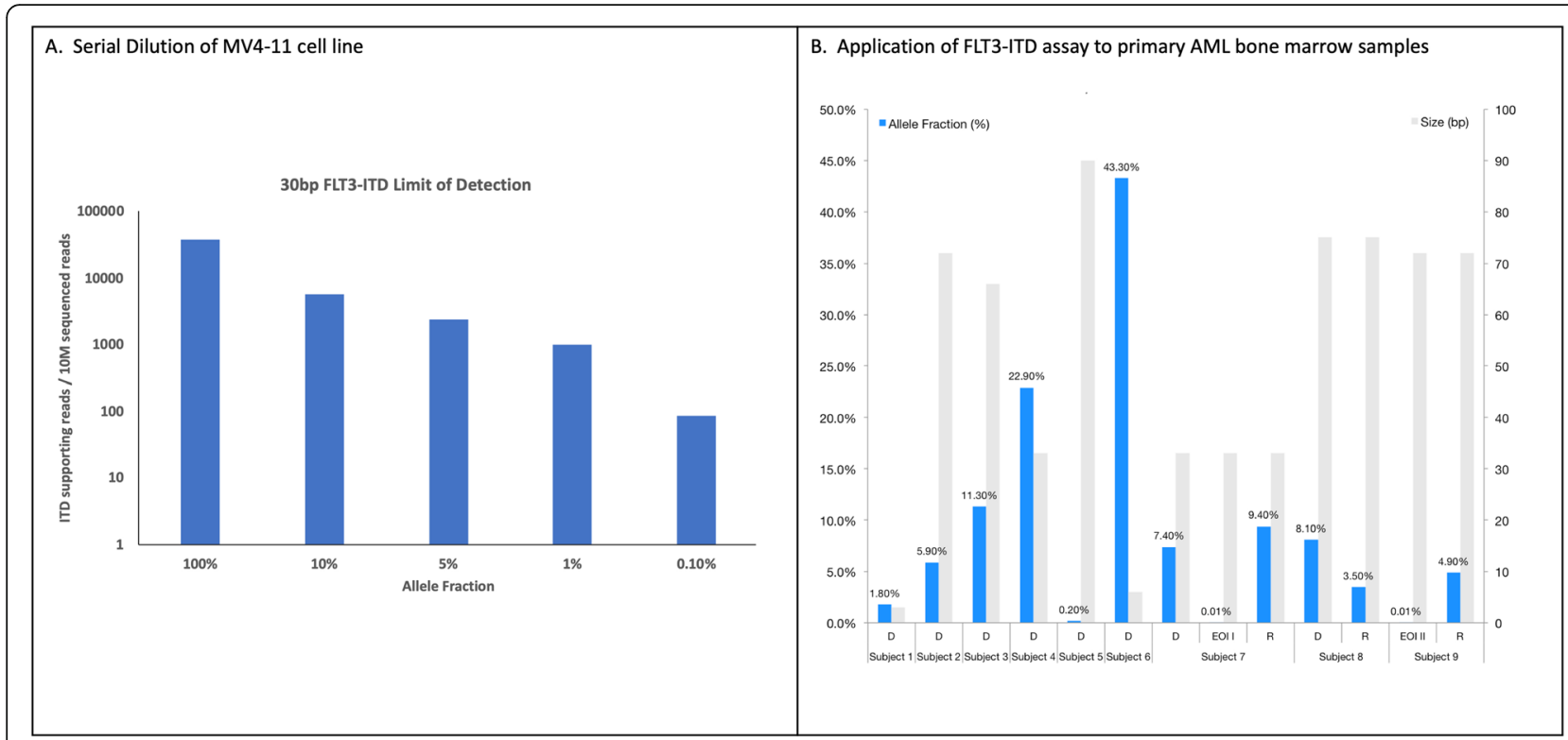

Fig. 3 Development of a specific and sensitive NGS panel for FLT3-ITD detection and disease monitoring. a Serial dilution results for MV4-11 cell line (30 bp ITD in FLT3). The $x$-axis is allele fraction and $y$-axis is the number of ITD supporting reads / $10 \mathrm{M}$ sequencing reads. $\mathbf{b}$ Nine leukemia samples were analyzed with varying ITD sizes (right $y$-axis, grey bars) and allele fraction (left $y$-axis, blue bars). Subjects are across the $x$-axis, with $\mathrm{D}=$ diagnosis, $\mathrm{EOI}=$ end of induction, and $\mathrm{R}=$ relapse sample

Additionally, by using the primer locations for all target genes, ECS coupled with AMP technology enables detection of copy number gains or losses. The computational pipeline identified a loss of the $C B L$ locus (E3 ubiquitin-protein ligase $\mathrm{CBL}$ ) in one child (Additional file 3: Figure S3). The deletion is smaller than would be detected by cytogenetics or SNP array, but large enough to cause loss of PCR amplification that could be interpreted as a false negative for variation if only the wild type allele is amplified. Overall, these results provide better resolution and increased breadth of detectable lesions to the standard assays, as the position and sequence, along with accurate quantitation, is accomplished in a single assay.

\section{Digital quantification of RNA transcripts}

By incorporating UMIs to the targeted enrichment of cDNA molecules prior to amplification and sequencing, the ECS-RNA eliminates the issue of duplication bias, thus enabling digital, rather than relative (such as transcriptome sequencing), quantification of gene expression. As a proof of principle, we surveyed for gene expression patterns in a healthy umbilical cord blood and a pediatric AML remission sample using a commercial product that targets 416 cancer-associated genes. RNA-ECS can quantitatively detect mRNA copy numbers as low as < 10 copies, which was validated by droplet digital PCR (Additional file 4: Figure S4).

\section{Characterization of diagnostic leukemia samples using error-correct sequencing techniques}

To demonstrate the utility of our ECS capture technique for the detection of novel complex RNA variants, 27 primary pediatric diagnostic samples were analyzed $(n=6$ AML; $n=1$ APL, $n=17$ preB-ALL, $n=3 \mathrm{~T}-\mathrm{ALL}$; Additional file 5: Table S1 demographics). Nine out of the 27 primary bone marrow samples contained gene fusions detected by routinely tested FISH probes for diagnostic purposes consisting of ETV6-RUNX1 ( $n=4$ subjects), $B C R-A B L 1$ ( $\mathrm{n}=1$ subject), TCF3-PBX1 ( $\mathrm{n}=1$ subject), $P M L-R A R A$ ( $\mathrm{n}=1$ subject) and KMT2A rearrangements ( $n=2$ subjects). ECS-RNA not only confirmed these gene fusions but also detected previously undetected cryptic gene fusions in 10 subjects that were negative for chromosomal rearrangements via FISH. Some of these cryptic gene fusions, including NUP98-NSD1, P2RY8-CRLF2 and $T C F 3-H L F$, are recurrently seen in pediatric leukemias and their prognostic significance has been demonstrated [9-11]. Other identified gene fusions, such as STIL-TAL1, have an unclear role in T-ALL biology (A.L. [7]). Additionally, we identified novel in-frame gene fusions SPTAN1-ABL1, SSBP2-CHD1, RUNX1-MKL1, RCAN1RUNX1, and P2RY8-ANKLE2 (Table 2), which were confirmed by Sanger sequencing. One subject with an ETV6-RUNX1 fusion detected by FISH, showed two additional cryptic gene fusions by RNA-ECS (Table 2).

The remaining 6 subjects did not harbor detectable gene fusions by RNA sequencing. Two preB-ALL 
samples showed deletion of IKZF1 exons 4-6 (Additional file 7: Table S3); which have been reported in $9 \%$ of B-ALL patients [12] and results in expression of the dominant negative form of the transcription factor IKAROS lacking DNA binding zinc finger motifs [13]. Four unique and previously unidentified gene fusions via karyotyping data at diagnosis were identified in primary AML samples, KMT2A-MLLT1, KMT2A-MLLT3, NUP98$N S D 1$ and the reciprocal NSD1-NUP98, and 5 exon duplication/deletions were identified in CEBPA and IRF4 (Table 2 and Additional file 7: Table S3). These results demonstrate the diversity of prognostic or therapeutically relevant somatic mutations inherent in leukemias, which are often undetectable by gold standard methods.

In total, 143 RNA StVs were identified (Fig. 4a and Additional file 7: Table S3). All samples showed alternative exon usage and domain duplications/deletions for several known oncogenes along with novel mutations. We observed exon duplications in MYC (AF range 0.130.88 ), $B C L 11 B$ (AF range 0.12-.22), CEBPA (AF range 0.10-0.79), and ZCCHC7 (AF range 0.11-0.83), while exon deletions were noted in IRF4 (AF range 0.12-0.55), IKZF1 (AF range 0.40-0.44) and SETD2 (AF range 0.11-0.21) (Fig. 4b and Additional file 7: Table S3).
Intron retention, a characteristic of cancer transcriptomes [14], was also observed in 31 genes across the various samples (Fig. 4b). Of interest, 15 out of 18 of the B-ALL samples had a retained intron in $\mathrm{ZCCHC7}$ involving intron 2 (Fig. 5a), which is in a region known for breakpoints in pediatric B-ALL [15]. Furthermore, a novel cryptic gene fusion was identified in one of T-ALL samples involving SPTAN1 and ABL1 (Fig. 5b).

In addition to the StVs, $170 \mathrm{SNVs}$ were called. Almost half of SNVs (47\%) were localized to the untranslated regions (UTR), with missense and synonymous mutations being the second $(26 \%)$ and third (17\%) most common SNVs, respectively (Fig. 4c). SNVs causing frameshift mutations were found in $5 \%$ of variants, while nonsense mutations were found in $1 \%$ variants. $4 \%$ of SNVs were detected in exon:intron splice junctions, and the majority of the coding variants were located within $\mathrm{NOTCH1}$ (Fig. 4d).

\section{Discussion}

The heterogeneity of clinically relevant, low VAF genetic variation in healthy individuals and leukemia patients is far more diverse than previously appreciated and not readily detectable by standard diagnostics or even "deep sequencing" without UMIs. The clinical significance of
(A)

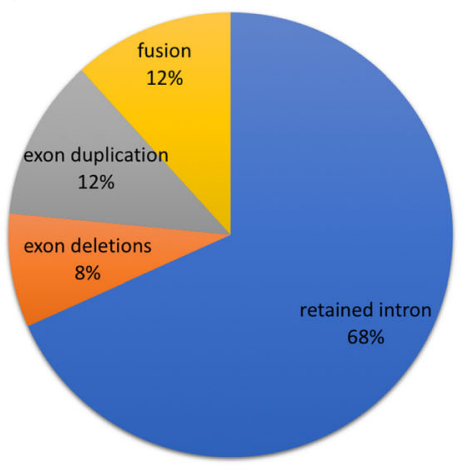

(C)

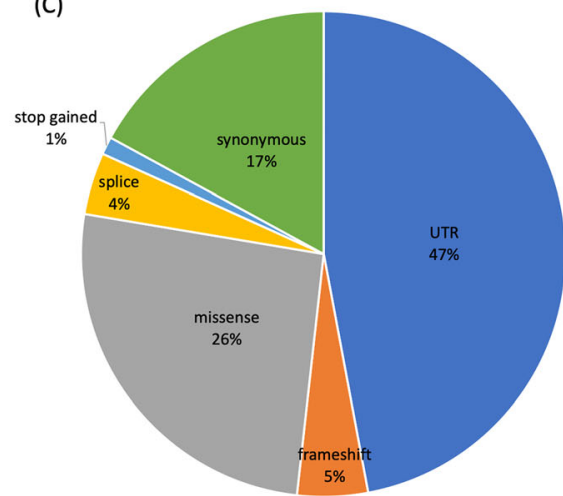

(B)

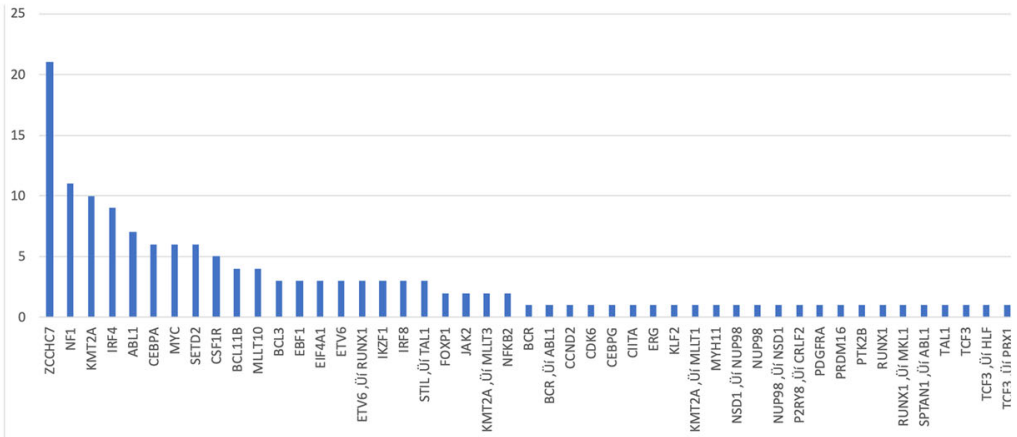

(D)

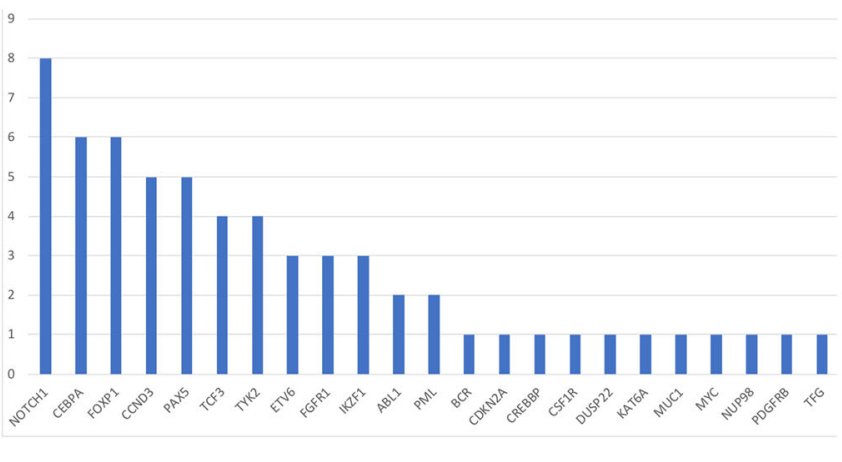

Fig. 4 Summary of RNA-ECS results for pediatric leukemia diagnostic samples. a Distribution of allelic specific single nucleotide variants and gene counts. Pie chart represents the distribution across all leukemia samples. b The bar graph represents the counts per gene. c Distribution of RNA StVs and gene counts. Pie chart represents the distribution across all leukemia samples for SNVs. $\mathbf{d}$ The bar graph represents the counts per gene 


\section{A. ZCCHC7 RNA splicing variant}

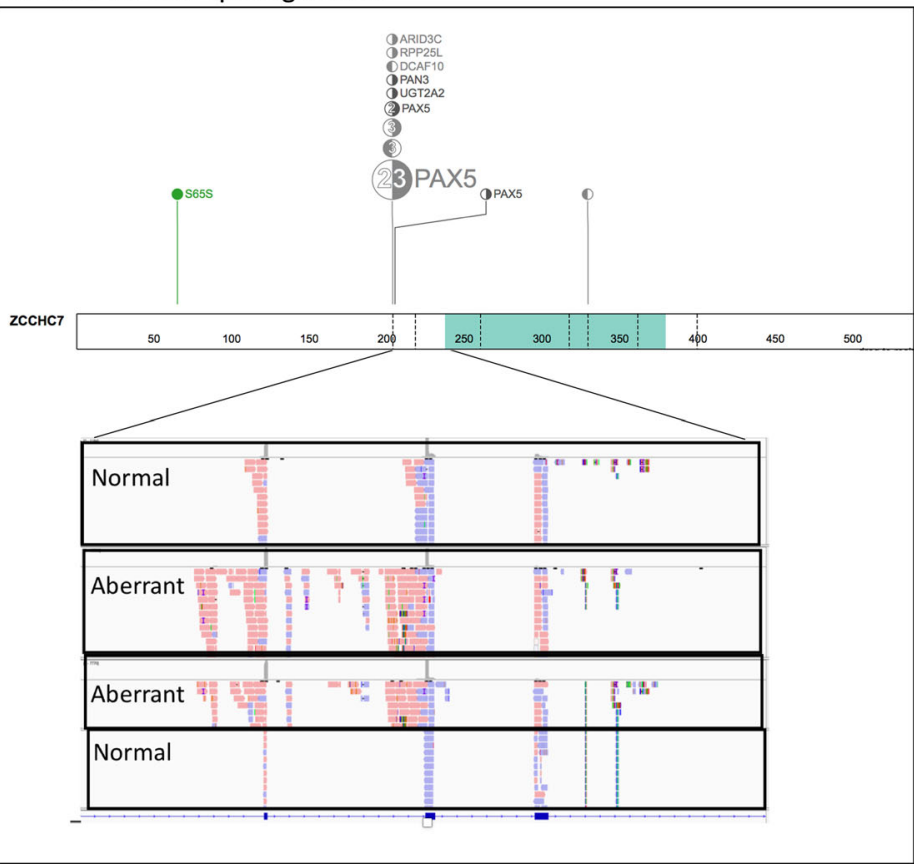

B. Novel cryptic gene fusion SPANT1 - ABL1

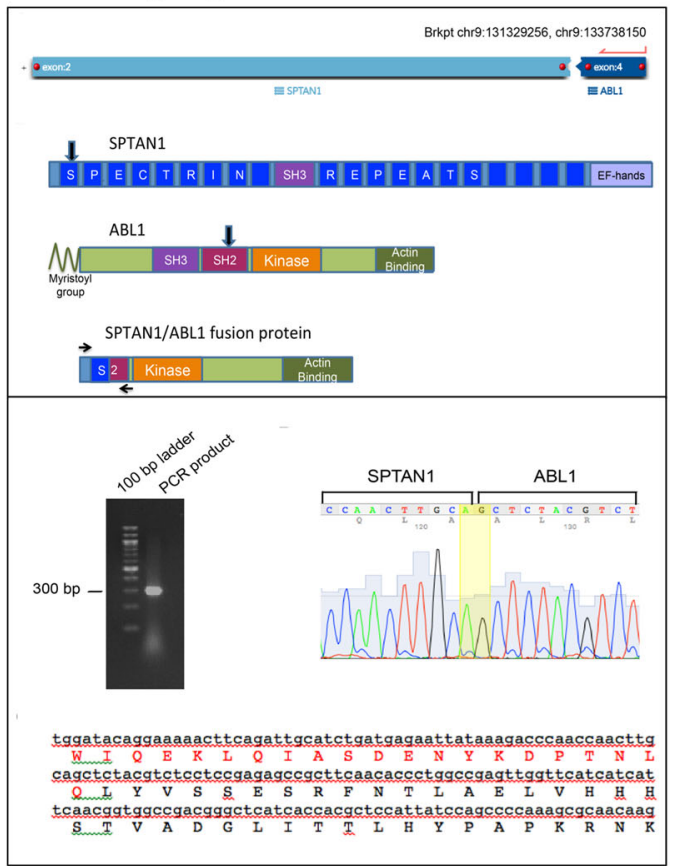

Fig. 5 Novel RNA variants identified at time of diagnosis. a An aberrant RNA molecule in ZCCHC7 was identified in several of the B-ALL samples. b Novel cryptic gene fusion in SPTAN1-ABL1 was identified in a T-ALL subject and confirmed via Sanger sequencing. ExPASy translation of the fusion product sequence revealed the in-frame fusion of SPTAN1 (amino acids in red) and ABL1 (amino acids in black)

this variation is only beginning to be understood, but as the repertoire of precision therapeutics in cancer expands, the optimization of risk prognostication and therapeutic selection requires precise quantification multiple cancerrelated mutations in DNA and RNA simultaneously at frequencies as low as 0.0001 , two orders of magnitude below the error rate of current NGS platforms.

This diversity requires a "toolbox" of sensitive and specific molecular and computational strategies for any leukemia, such as we have outlined here. The implementation of a molecule-specific, random indexing to enables the computational removal of stochastic sequencing and PCR errors to accurately call mutations at very low levels. These strategies can be used for clonal profiling and disease surveillance across a wide variety of targets simultaneously. The limit of detection established in this report are equivalent to the current flow-based methods and, as demonstrated, ECS is capable of overcoming many limitations of traditional NGS assays [14]. For instance, while a clinical molecular MRD assay does exist for FLT3-ITD (Lin et al., 2015), it is only a single mutation assay and is not capable of detecting clinically relevant co-occurring mutations. Furthermore, the detection of ITDs $>80 \mathrm{bp}$ has historically been quite difficult with anything other than whole genome sequencing, including hybridization methods [16].
Another interesting observation is the various types of hematopoietic clones apparent in a single individual at different points in time during therapy. We intended to characterize the disappearance of AML-associated mutations from diagnosis to EOI and how many of these clonal mutations reappeared in those that relapsed. However, we find various classes of mutations at each time point. In addition to the expected loss of AML-related clonal mutations, we see mutations that are extremely stable at each time point, apparently unaffected by cytotoxic chemotherapy, but unrelated to the patient's leukemia.

The number of clonal mutations gained after induction therapy is quite similar to the number of clones that disappear after induction chemotherapy, resulting in a roughly stable absolute number of hematopoietic clones, focusing relevance on the nature of the mutation rather than the abundance. Finally, there are some clones that actually appear to increase after induction therapy, suggesting they have a growth advantage, which is akin to prior reports of pre-existing TP53-mutated hematopoietic clones that expand following chemotherapy for a primary malignancy, but sometimes acquire additional mutations that result in transformation to therapy-related AML [17]. Of these, mutations in IKZF1 were the most common to demonstrate an increased VAF at EOI for several of the subjects, and several of the subjects had a deletion as 
similar described by de Rooij et al. [18]. These observations must be weighed against the small sample size and the fact that this sequencing panel was limited in breadth.

\section{Conclusion}

ECS coupled with an AMP approach enables the detection of complex low allelic variants. This technique has the potential to further advance our understanding of MRD and personalized medicine. Further studies utilizing single cell DNA or RNA sequencing that can quantify allele-specific expression would provide important additional insights for mutation co-localization and associated impacts on gene expression and cellular phenotype. While future ECS-based studies could provide better resolution than current flow-based MRD assays, correlating identified mutations with true disease, risk stratification and therapeutic selection will require much larger, well phenotype studies.

\section{Supplementary information}

Supplementary information accompanies this paper at https://doi.org/10. 1186/s12920-020-0671-8.

\begin{abstract}
Additional file 1: Figure S1. Bioinformatics utilities for variant detection. The workflow described consists of 3 major areas: capture technique, alignment method, and variant detection. In this report we focused on AMP and amplicon based technologies. Two different alignment methods were used: force reference alignment and de novo assembly. Multiple methods were used for variant detection including freebayes, LoFreq, and ARBA. The results from the various algorithms are ultimately merged and displayed in our custom graphical interface.

Additional file 2: Figure S2. Schema of error-corrected sequencing molecules. Library preparation for ECS includes the addition of a molecular barcode (dark grey box) that enables identification of molecular bins. Each subject is given a sample index (green box) during library preparation which allows multiple samples to be pooled during sequencing. Each molecule contains a universal primer site (purple box) and a random adaptor ligation (yellow box).
\end{abstract}

Additional file 3: Figure S3. Graphical representation of a CNV loss in CBL. Each of the dots represents a gene specific primer (GSP). Deviation from 2 copies are represent below the line (loss) or above (gain).

Additional file 4: Figure S4. RNA-ECS is accurate to single transcripts without normalization. (A and B) Technical replicates from umbilical cord blood and a pediatric AML remission bone marrow aspirate. Absolute numbers of transcripts for all genes with fewer than 100 called copies are plotted (genes with $>100$ copies were not included on the plot to highlight limit of detection) showing strong technical replication. (C) Transcript counts spanning two orders of magnitude were validated via $\mathrm{ddPCR}$, showing a strong concordance.

Additional file 5: Table S1. Summary of demographics for the pediatric leukemia samples.

Additional file 6: Table S2. Genes targeted on the DNA and RNA panels. Additional file 7: Table S3. Structural variants identified via RNA-ECS in primary diagnostic samples.

\section{Abbreviations}

ALL: Acute lymphoblastic leukemia; AML: Acute myelogenous leukemia; AMP: Anchored multiplexed PCR; APL: Acute promyelocytic leukemia; CNV: Copy number variation; COG: Children's Oncology Group; ECS: Errorcorrected sequencing; FISH: Fluorescence in situ hybridization; GSPs: Genespecific primers; ITD: Internal tandem duplication; LAIP: Leukemia-associated immunophenotype; MPFC: Multi-parameter flow cytometry; MRD: Minimal residual disease; NGS: Next generation sequencing; SNVs: Single nucleotide variants; StVs: Structural variants; TARGET: Therapeutically Applicable Research to Generate Effective Treatments; UMI: Unique molecular index; UTR: Untranslated regions; VAF: Variant allele frequency

\section{Acknowledgements}

We thank our funding agencies (NIH, Hyundai, and LRFDE) for supporting this work; the Nemours Center for Caner and Blood Disorders for supporting $\mathrm{ELC}, \mathrm{SB}, \mathrm{AG}$, and $\mathrm{AK}$.

Ethics and approval and consent to participate

All samples used in this study were collected from human peripheral blood or bone marrow. Specimens were collected for biobanking and subsequent de-identified biomedical research at Nemours following written informed consent (Human Research Protection Office (HRPO) IRB\# 349465), including parental permission forms (child/adolescent assent was obtained for ages 717 years). The Nemours samples used in this study were deemed nonhuman research and approved by the Nemours / A.l. duPont Children's Hospital (HRPO IRB\# 267207). Patients enrolled on the Children's Oncology Group (COG) AAML1031 phase III pediatric de novo AML study (NCT00372593) had the option to consent/assent for correlative biomedical research when enrolling for the study. All available COG samples were deidentified and personal health information (PHI) was not available to study team. Adult AML specimens are banked at Washington University for biomedical research under Human Research Protection Office (HRPO) IRB\# 201011766. This study was approved as by the Washington University HRPO IRB\# 201511125 entitled "Error-corrected sequencing for minimal residual disease in AML.

\section{Availability of data materials}

The data for this study are available via the European Nucleotide Archive (ENA) Accession PRJEB36035.

\section{Authors' contributions}

TD conceived the study and participated in its design and coordination. NM, $\mathrm{SB}, \mathrm{AG}$, and WHW were responsible for all of the wet-bench work, and ELC was responsible for all of the data analytics and bioinformatics analysis. EAK provided guidance for the data analysis (ELC) and the project. All authors worked on drafting and revising the manuscript, and all authors read and approved the final manuscript.

\section{Funding}

NIH NCl R01CA211711 (PI Druley); Siteman Cancer Center Research Fund 13FY16-01 (PI Druley); Hyundai Hope on Wheels 2015Q3-3 (PI Druley); Children's Discovery Institute MC-II-2015-461 (PI Druley); Leukemia Research Foundation of Delaware (PI Kolb).

\section{Consent for publication}

Not applicable.

\section{Competing interests}

The authors declare that they have no competing interests.

\section{Author details}

'Biomedical Research Department, Nemours / A.l. DuPont Children's Hospital, Wilmington, DE, USA. ${ }^{2}$ Department of Pediatrics, Washington University School of Medicine, 660 South Euclid Avenue, Campus Box 8116, St. Louis, MO 63110, USA. ${ }^{3}$ Edison Family Center for Genome Sciences and Systems Biology, Washington University School of Medicine, 4515 McKinley Avenue, Campus Box 8510, St. Louis, MO 63108, USA. ${ }^{4}$ Nemours Center for Cancer and Blood Disorders, Nemours/A.I. duPont Hospital for Children, Wilmington, USA.

Received: 26 April 2019 Accepted: 24 January 2020

Published online: 04 March 2020

\section{References}

1. Yin JA, O'Brien MA, Hills RK, Daly SB, Wheatley K, Burnett AK. Minimal residual disease monitoring by RT-qPCR in core-binding factor AML allows risk-stratification and predicts relapse: results of the UK MRC AML-15 trial. Blood. 2012;120(14):2826-35. https://doi.org/10.1182/blood-2012-06-435669. 
2. Borowitz MJ, Devidas M, Hunger SP, Bowman WP, Carroll AJ, Carroll WL, Camitta BM. Clinical significance of minimal residual disease in childhood acute lymphoblastic leukemia and its relationship to other prognostic factors: a Children's oncology group study. Blood. 2008;111(12):5477-85. https://doi.org/10.1182/blood-2008-01-132837.

3. Borowitz MJ, Wood BL, Devidas M, Loh ML, Raetz EA, Salzer WL, Larsen E. Prognostic significance of minimal residual disease in high risk B-ALL: a report from Children's oncology group study AALL0232. Blood. 2015;126(8): 964-71. https://doi.org/10.1182/blood-2015-03-633685.

4. Loken MR, Alonzo T a, Pardo L, Gerbing RB, Raimondi SC, Hirsch B a, Meshinchi S. Residual disease detected by multidimensional flow cytometry signifies high relapse risk in patients with de novo acute myeloid leukemia: A report from Children's oncology group. Blood. 2012;120(8):1581-8. https:// doi.org/10.1182/blood-2012-02-408336.

5. van der Velden VHJ, van der Sluijs-Geling A, Gibson BES, te Marvelde JG, Hoogeveen PG, Hop WCJ, van Dongen JM. Clinical significance of flowcytometric minimal residual disease detection in pediatric acute myeloid leukemia patients treated according to the DCOG ANLL97/MRC AML12 protocol. Leukemia. 2010;24(9):1599-606. https://doi.org/10.1038/leu.2010.153.

6. Young AL, Challen GA, Birmann BM, Druley TE. Clonal haematopoiesis harbouring AML-associated mutations is ubiquitous in healthy adults. Nat Commun. 2016;7:12484. https://doi.org/10.1038/ncomms12484.

7. Young AL, Wong TN, Hughes AEO, Heath SE, Ley TJ, Link DC, Druley TE. Quantifying ultra-rare pre-leukemic clones via targeted error-corrected sequencing. Leukemia. 2015;29(7):1608-11. https://doi.org/10.1038/leu.2015.17.

8. Bolouri H, Farrar JE, Triche T, Ries RE, Lim EL, Alonzo TA, et al. The molecular landscape of pediatric acute myeloid leukemia reveals recurrent structural alterations and age-specific mutational interactions. Nat Med. 2018;24(1): 103-12. https://doi.org/10.1038/nm.4439.

9. Dou H, Chen X, Huang Y, Su Y, Lu L, Yu J, et al. Prognostic significance of P2RY8-CRLF2 and CRLF2 overexpression may vary across risk subgroups of childhood B-cell acute lymphoblastic leukemia. Genes, Chromosomes Cancer. 2017;56(2):135-46. https://doi.org/10.1002/gcc.22421.

10. Fischer U, Forster M, Rinaldi A, Risch T, Sungalee S, Warnatz H-J, Yaspo M-L. Genomics and drug profiling of fatal TCF3-HLF-positive acute lymphoblastic leukemia identifies recurrent mutation patterns and therapeutic options. Nat Genet. 2015;47(9):1020-9. https://doi.org/10.1038/ng.3362.

11. Ostronoff F, Othus M, Gerbing RB, Loken MR, Raimondi SC, Hirsch BA, Meshinchi S. NUP98/NSD1 and FLT3/ITD coexpression is more prevalent in younger AML patients and leads to induction failure: a COG and SWOG report. Blood. 2014;124(15):2400-7. https://doi.org/10.1182/blood-2014-04-570929.

12. Churpek JE, Pyrtel K, Kanchi K-L, Shao J, Koboldt D, Miller CA, Graubert TA. Genomic analysis of germ line and somatic variants in familial myelodysplasia/acute myeloid leukemia. Blood. 2015;126(22):2484-90. https://doi.org/10.1182/blood-2015-04-641100.

13. Jongen-Lavrencic M, Grob T, Hanekamp D, Kavelaars FG, al Hinai A, Zeilemaker A, Valk PJM. Molecular Minimal Residual Disease in Acute Myeloid Leukemia. N Engl J Med. 2018;378(13):1189-99. https://doi.org/10. 1056/NEJMoa1716863.

14. Salk JJ, Schmitt MW, Loeb LA. Enhancing the accuracy of next-generation sequencing for detecting rare and subclonal mutations. Nat Rev Genet. 2018;19(5):269-85. https://doi.org/10.1038/nrg.2017.117.

15. Heinäniemi M, Vuorenmaa T, Teppo S, Kaikkonen MU, Bouvy-Liivrand M, Mehtonen J, Lohi O. Transcription-coupled genetic instability marks acute lymphoblastic leukemia structural variation hotspots. ELife. 2016;5. https:// doi.org/10.7554/eLife.13087.

16. Crowgey EL, Kolb A, Wu CH. Development of Bioinformatics Pipeline for Analyzing Clinical Pediatric NGS Data. In: AMIA Joint Summits on Translational Science Proceedings AMIA Summit on Translational Science, 2015; 2015. p. 207-11. Retrieved from http://www.ncbi.n/m.nih.gov/ pubmed/26306272.

17. Wong TN, Ramsingh G, Young AL, Miller CA, Touma W, Welch JS, et al. Role of TP53 mutations in the origin and evolution of therapy-related acute myeloid leukaemia. Nature. 2014;518(7540):552-5. https://doi.org/10.1038/ nature13968.

18. de Rooij JDE, Beuling E, van den Heuvel-Eibrink MM, Obulkasim A, Baruchel A, Trka J, et al. Recurrent deletions of IKZF1 in pediatric acute myeloid leukemia. Haematologica. 2015;100(9):1151-9. https://doi.org/10.3324/ haematol.2015.124321

\section{Publisher's Note}

Springer Nature remains neutral with regard to jurisdictional claims in published maps and institutional affiliations.
Ready to submit your research? Choose BMC and benefit from:

- fast, convenient online submission

- thorough peer review by experienced researchers in your field

- rapid publication on acceptance

- support for research data, including large and complex data types

- gold Open Access which fosters wider collaboration and increased citations

- maximum visibility for your research: over $100 \mathrm{M}$ website views per year

At BMC, research is always in progress.

Learn more biomedcentral.com/submissions 